\title{
Atomic-Resolution Monitoring of Structural Phase Transition in Bi-magnetic Core/Shell Oxide Nanoparticles
}

\author{
M.A. Roldan ${ }^{1,2}$, J. Salafranca ${ }^{1,2}$, R. Ishikawa $^{2}$, R. Mishra ${ }^{3}$, A. Lopez-Ortega ${ }^{4}$, M. Estrader ${ }^{5}$, \\ G. Salazar-Alvarez ${ }^{6}$, J. Nogues. ${ }^{7,8}$, S. J. Pennycook ${ }^{9}$ and M. Varela ${ }^{2,1}$ \\ 1. Universidad Complutense de Madrid, Madrid 28040, Spain. \\ 2. Oak Ridge National Laboratory, Oak Ridge TN 37831, USA. \\ 3. Dept. of Physics and Astronomy, Vanderbilt University, Nashville, TN 37235, USA. \\ 4. INSTM and Dept. di Chimica “U. Schiff”, Universitá degli Studi di Firenze, Firenze 50121, Italy. \\ 5. Depto. de Química Inorgánica, Universitat de Barcelona, Barcelona 08028, Spain. \\ 6. Department of Materials and Enviromental Chemistry, Stockholm University, Stockholm 11418, \\ Sweden \\ 7. ICN2-Institut Catala de Nanociencia i Nanotechnologia, Campus UAB, Bellaterra 08193, Spain. \\ 8. ICREA, Barcelona 08010, Spain. \\ 9 University of Tennessee, Knoxville, Tennessee 37996, USA.
}

Transition metal oxide nanoparticles (NP) often exhibit properties not present in bulk. The low dimensionality on the one hand, and structural and chemical inhomogeneities on the other, may severely affect the system's properties and their magnetic response. In particular, exchange coupling in bi-magnetic core/shell nanoparticles has attracted considerable attention since the presence of the interface may result in novel behaviors [1]. In order to harness the properties of complex oxide nanosystems, probes that can analyze structural and chemical homogeneity with high spatial resolution are essential. Aberration corrected scanning transmission electron microscopy combined with electron energy loss spectroscopy (STEM-EELS) and theoretical calculations can achieve these goals [2].

In the present work we study bi-magnetic Fe3O4/Mn3O4 core/shell nanoparticles at the atomic scale by STEM-EELS. Two different kind of samples were studied, a first set with iron oxide in the core and the manganese oxide in the shell and a second set of samples with the opposite configuration. Different acceleration voltages $(60,100$ and $200 \mathrm{kV})$ were used in order to probe any electron beam induced damage. High resolution STEM images, such as the high angle annular dark field (HAADF) image of a $\mathrm{Fe}_{3} \mathrm{O}_{4} / \mathrm{Mn}_{3} \mathrm{O}_{4}$ core/shell NP shown in Figure 1(a), exhibit a very high structural quality as well as a coherent interface between the shell and core [1]. Figure 1 also shows EELS maps extracted from the Fe $\mathrm{L}_{2,3}$ edge (red) and $\mathrm{Mn} \mathrm{L}_{2,3}$ (green) respectively, together with an oxygen content map and values obtained along the yellow arrow. Quantification of the EELS data acquired at $60 \mathrm{kV}$ shows that the chemical composition of the nanoparticles is indeed Fe3O4 and Mn3O4.

Observation at $200 \mathrm{kV}$, however, results in a partial reduction of the $\mathrm{Mn} 3 \mathrm{O} 4$ to $\mathrm{MnO}$. Figure 2 displays the result of a spectrum image with these two phases in the shell. To quantify the distribution of the different oxides detected we performed multiple linear least squares (MLLS) fitting routines on our spectrum images. The transition from $\mathrm{Mn} 3 \mathrm{O} 4$ to $\mathrm{MnO}$ is produced by the electron beam at $200 \mathrm{kV}$ $\left(\mathrm{Fe}_{3} \mathrm{O}_{4}\right.$ does not exhibit major signs of damage). Here, we will demonstrate how the atomic-resolution phase transition that takes place under the beam can be studied using the particle image velocimetry (PIV) [3]. PIV measures whole velocity fields by taking two atomic resolution images acquired shortly after each other and calculating the distance of individual atoms travelled within this time. We will show the possibility to track atoms and trace their path while the transformation is taking progress. Using PIV, we will show how the reaction progresses. We will also show how the resulting structural changes result 
in modifications in the dimensions of the nanoparticles. These dimensional modifications stabilize the transformed nanoparticles. Theoretical calculations will be also used to gain insights about the origin of the material-selective reduction.

\section{References:}

[1] M. Estrader et al. Nat. Commun. 4 (2013) 2960.

[2] M. Varela et al. Annu. Rev. Mater. Res. 35 (2005) p. 539.

[3] Tseng Q. et al. PNAS 109 (2011) p. 1506.

[4] H. Kurata and C. Colliex. Phys. Rev. B 48 (1993) p. 2102.

[5] Research supported by the European Research Council Starting Investigator Award STEMOX \# 239739 (M.R. and J. S.), JSPS Postdoctoral Fellowship for Research Abroad (R.I.) by the U.S.

Department of Energy (DOE), Basic Energy Sciences (BES), Materials Sciences and Engineering Division (RM, SJP, MV) and through a user project supported by ORNL's Center for Nanophase Materials Sciences (CNMS), which is sponsored by the Scientific User Facilities Division, Office of Basic Energy Sciences, U.S. Department of Energy.
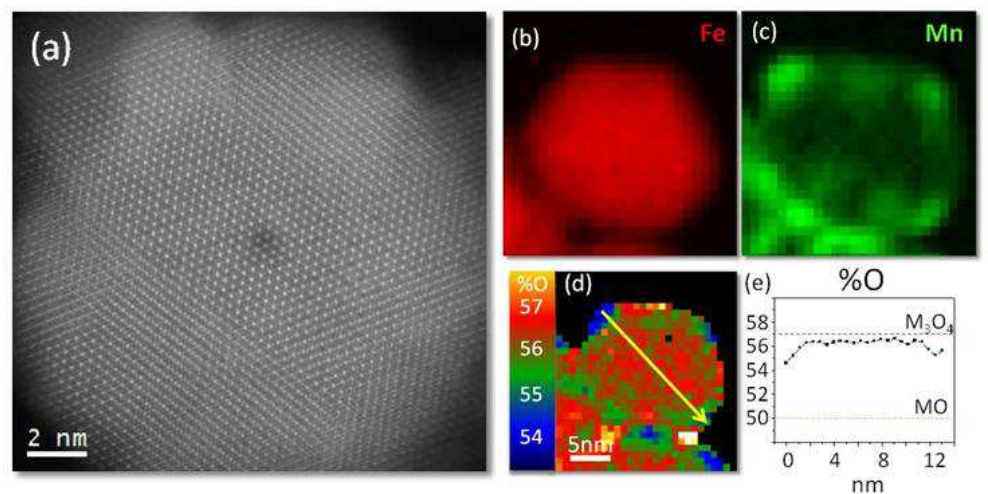

Figure 1. (a) HAADF image of a $\mathrm{Fe}_{3} \mathrm{O}_{4} / \mathrm{Mn}_{3} \mathrm{O}_{4}$ Core/Shell nanoparticle. EELS maps for a Core/Shell nanoparticle obtained at $60 \mathrm{kV}$ in a Nion UltraSTEM extracted from the (b) Fe $\mathrm{L}_{2,3}$ edge (red) and (c) $\mathrm{Mn} \mathrm{L}_{2,3}$ (green) respectively. Oxygen content map (d) and values (e) obtained along the yellow arrow. EEL Spectrum image were recorded at $0.2 \mathrm{~s} / \mathrm{pixel}$ with an energy dispersion of $0.25 \mathrm{eV} / \mathrm{channel}$.
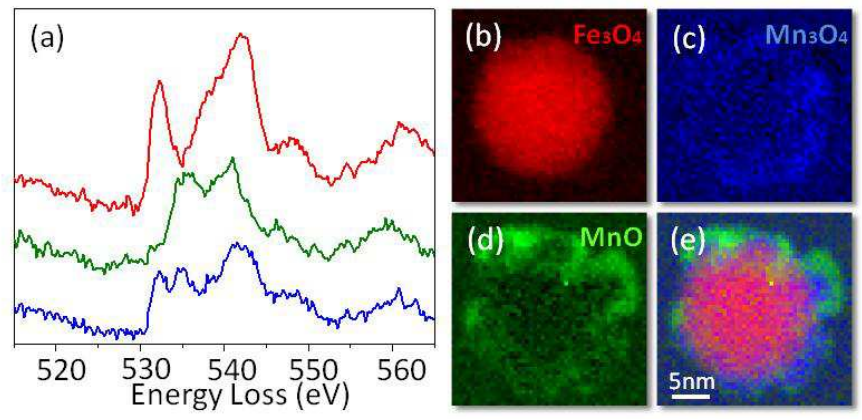

Figure 2. (a) ELNES of the $\mathrm{O}-\mathrm{K}$ edge measured on the $\mathrm{Fe}_{3} \mathrm{O}_{4}$ core (red), MnO-like nanodomains (green) and Mn3O4- like areas (blue), consistent with bulk spectra [4]. These components were used as reference spectra for the MLLS maps of a $\mathrm{Fe}_{3} \mathrm{O}_{4} / \mathrm{Mn}_{3} \mathrm{O}_{4}$ Core/Shell nanoparticle obtained at $200 \mathrm{kV}$ for (b) $\mathrm{Fe}_{3} \mathrm{O}_{4}$ (c) $\mathrm{Mn}_{3} \mathrm{O}_{4}$ and (d) $\mathrm{MnO}$ respectively. (e) Corresponding RGB colored map, with $\mathrm{Fe}_{3} \mathrm{O}_{4}$ in red, $\mathrm{MnO}$ in green, and $\mathrm{Mn}_{3} \mathrm{O}_{4}$ in blue. EEL spectrum image recorded at $0.1 \mathrm{~s} /$ pixel with an energy dispersion of $0.3 \mathrm{eV} /$ channel. 